\title{
Recursos vegetais usados por Acromyrmex striatus (Roger) (Hymenoptera, Formicidae) em restinga da Praia da Joaquina, Florianópolis, Santa Catarina, Brasil
}

\author{
Benedito C. Lopes \\ Departamento de Ecologia e Zoologia, Universidade Federal de Santa Catarina. Caixa Postal 476, 88010-970 Florianópolis, \\ Santa Catarina, Brasil.E-mail: bclopes@ccb.ufsc.br
}

\begin{abstract}
Vegetable resources used by Acromyrmex striatus (Roger) (Hymenoptera, Formicidae) in sand dunes at Joaquina Beach, Florianópolis, Santa Catarina State, Brazil. A total of 400 nests of Acromyrmex striatus (Roger, 1863) was evaluated between 1997 and 1998 at the dunes of the Joaquina Beach, Florianópolis, Santa Catarina State, in order to determine the vegetable substrate brought back to the nests. These ants use some parts of 50 plant species in 22 botanical families, being Compositae, Gramineae and Leguminosae the principal ones. In this last family, Stylosanthes viscosa Swartz was the most herbivored species in the two years samplings. Acromyrmex striatus cut fresh vegetables, as well as use decayed materials, being then a true leaf-cutter ant or a opportunistic ant.

KEY WORDS. Leaf-cutter ant, resources, sand dunes, southern Brazil.
\end{abstract}

RESUMO. Foram amostrados 400 ninhos de Acromyrmex striatus entre 1997 e 1998, nas dunas da praia da Joaquina, Florianópolis, SC, para a verificação do material vegetal trazido para o ninho. Estas formigas usam partes de 50 espécies de plantas dispostas em 22 famílias, sendo as principais, representantes de Compositae, Gramineae e Leguminosae. Nesta última família, Stylosanthes viscosa foi a espécie mais utilizada nos dois anos de amostragem. Acromyrmex striatus corta matéria vegetal fresca, bem como se utiliza de material vegetal já caído, podendo, então se comportar como cortadeira ou como uma espécie oportunista.

PALAVRAS CHAVE. Formiga cortadeira, recursos vegetais, restinga, sul do Brasil.

A cultura do fungo, que servirá principalmente à alimentação das larvas, é feita pelas operárias de cortadeiras às custas de uma grande gama de substratos recolhidos e trazidos até o ninho. Estes recursos variam desde fezes e carcaças de artrópodes até material vegetal fresco, cortado diretamente das plantas (Weber 1972, Hölldobler \& Wilson 1990).

Grande parte dos estudos de uso de recursos provêm da observação e registro das atividades de corte de espécies de Acromyrmex Mayr, 1865 e Atta Fabricius, 1804 em vegetação de interesse econômico (Fowler et al. 1986). Mais recentemente, tem havido uma tendência de considerar as formigas cortadeiras como "não pragas" ou até mesmo como benéficas em ambientes naturais, ao promoverem a reciclagem do solo e modificarem as características das comunidades vegetais onde se encontram as colônias (Fowler et al. 1989, DieHl-Fleig 1995, FARJIBrener \& Silva 1995, Vasconcelos \& Cherrett 1997, Farji-Brener \& Ghermandi 2000, Farji-Brener \& Illes 2000, Moutinho et al. 2003, VеRCHOT et al. 2003).

Os critérios de escolha e corte da vegetação por parte das operárias de Acromyrmex e Atta também têm sido a razão de muitos estudos. HowARD (1991) sintetiza três características do forrageamento de cortadeiras que sempre intrigaram os pes- quisadores: a) amostragem de numerosas espécies de plantas pelas operárias e preferência por poucas destas espécies; b) abandono de indivíduos de plantas muito preferidas, mesmo antes destes terem sido completamente desfolhados e c) escolha de indivíduos de plantas a longas distâncias do ninho e o não forrageamento sobre a mesma espécie, muitas vezes localizada bem mais próxima à colônia.

Três modelos ou hipóteses têm tentado explicar os padrões de forrageamento das cortadeiras. O mais antigo, o da "estratégia de conservação de recursos" ("conservational grazing system", Cherretr 1968), sugere que as operárias escolheriam forragear sobre plantas mais distantes do ninho como uma maneira de evitar a super-exploração da mesma espécie junto ao ninho, e se comportando, portanto, como "herbívoros prudentes". Um modelo alternativo, o do "forrageamento ótimo" ("optimal foraging") foi proposto por Fowler \& STILES (1980). Esta hipótese sugere que os padrões observados de forrageamento visam maximizar a qualidade do material coletado. As operárias, usando um sistema de trilhas, procuram por indivíduos vegetais que forneçam melhor qualidade, mesmo que estes estejam distantes do ninho. Uma terceira hipótese, proposta por Howard (1990) é a do "balanço entre nutrientes e 
aleloquímicos" ("nutrient/alelochemical balancing") que estabelece haver um controle das plantas a serem forrageadas, de maneira a fornecer os nutrientes necessários ao crescimento do fungo, bem como evitar substâncias tóxicas possivelmente presentes nestas plantas.

A escolha de recursos pelas operárias de cortadeiras também passa pelas características físicas e químicas das plantas a serem forrageadas.

No que se refere aos aspectos físicos, as principais características que influenciam na seleção parecem ser a textura, a pilosidade e a dureza do material vegetal, folha ou não (CHERRETT 1972, Waller 1982a, b, Hubbell et al. 1984).

Quanto aos aspectos químicos, deve-se destacar a importância positiva de alguns elementos como nitrogênio, fósforo, manganês e alumínio, a quantidade e qualidade de proteínas, carboidratos e lipídios, quantidade de água, presença e concentração de substâncias do metabolismos secundário das plantas, que podem agir como atraentes ou deterrentes do forrageamento (Bowers \& Porter 1981, Hubbell et al. 1984, Berish 1986, Howard 1988, 1990, Fowler \& Schlindwein 1994, Sugayama \& Salatino 1995, Vasconcelos \& Cherrett 1996).

A presença de látex em algumas espécies de plantas pode ser outro fator limitante ao ataque pelas cortadeiras. Concordando com JANZEN (1980), de que o látex pode funcionar mais como barreira física do que química, STRADLING (1978) registrou que operárias de Atta cephalotes (Linnaeus, 1758) cortam folhas secas e não folhas verdes de Euphorbia leucocephala Lotsy, uma espécie laticífera.

Os objetivos do presente trabalho foram: a) verificar, em termos qualitativos e quantitativos, os recursos vegetais utilizados para a fungicultura por operárias de Acromyrmex striatus (Roger, 1863) e b) verificar se há variações sazonais no uso destes recursos.

\section{MATERIAL E MÉTODOS}

\section{Área de estudo}

O estudo foi realizado na restinga da Praia da Joaquina (273' $40^{\prime \prime}$ S e $\left.48^{\circ} 27^{\prime} 10^{\prime \prime} \mathrm{W}\right)$, Florianópolis, Santa Catarina. Esta praia, situada no setor sudoeste da Ilha de Santa Catarina tem um comprimento de cerca de $3 \mathrm{Km}$ e apresenta, do mar para o interior, uma série de dunas fixas cobertas por vegetação arbustiva e herbácea, seguida de baixadas onde se encontram lagos permanentes e depressões úmidas, circundadas por dunas de pequeno porte (CASTELlani et al. 1996).

\section{Espécie em estudo}

Acromyrmex striatus é uma espécie de cortadeira polimórfica, com três tamanhos diferentes para as operárias (as maiores com 6,5 mm de comprimento), facilmente caracterizada dentro do gênero, pelo gáster liso e brilhante, sem tubérculos, além da presença de estrias longitudinais na cabeça. O alitronco e a cabeça são avermelhados ou ferruginosos, enquanto que o gáster é mais escuro, quase preto (GonÇALVEs 1961).
Esta espécie ocorre na Argentina, Bolívia, Brasil (apenas em Santa Catarina e Rio Grande do Sul), Paraguai e Uruguai (Gonçalves 1961, Fowler 1979a).

Os ninhos de Acromyrmex striatus ocorrem principalmente em locais abertos, com uma região limpa ao redor das aberturas, geralmente sem a formação de crateras ou montes de terra escavada (Diehl-Fleig 1995).

\section{Uso de recursos vegetais por Acromyrmex striatus}

Foram analisados 400 ninhos de Acromyrmex striatus, entre 1997 e 1998, 50 ninhos por estação do ano, com tempo de observação variando de 10 minutos (poucas operárias em forrageamento, com uma ou duas trilhas curtas) até 40 minutos (muitas operárias em forrageamento, com duas ou mais trilhas longas). Para cada ninho era feita a identificação do material trazido, acompanhando-se as operárias até os vegetais sendo cortados e/ou recolhidos. Em cada caso eram registrados: a espécie da planta, a altura, o estado fenológico e as partes do vegetal sendo herbivorado (folha, ramo, flor ou fruto); também, as operárias eram acompanhadas nas trilhas para o registro do material vegetal seco sendo recolhido.

Além dessas observações padronizadas, há poucos registros de outras plantas sendo usadas, em observações esporádicas.

Para a comparação dos recursos, a nível sazonal, foram usados os índices de diversidade de Simpson (cada listagem de material usado por estação é considerada uma agregação ou comunidade diferente) e o de similaridade de Morisita. Para avaliar a significância dos valores obtidos com o índice de Simpson, foi aplicado o teste $t$, conforme procedimentos em Brower et al. (1998). Também foi usado o teste G para avaliar diferenças na ocorrência dos itens utilizados pelas operárias de Acromyrmex striatus (BROWER et al. 1998).

\section{RESULTADOS}

Dos 400 ninhos observados, foi verificado o forrageamento em 128 dos 200 do ano de 1997 e em 139 dos 200 de 1998.

As operárias de Acromyrmex striatus utilizaram 50 espécies de plantas de 22 famílias, além de pedaços de líquens, fezes de lagartas de Lepidoptera e "material vegetal não identificado" (Tab. I). Nesta tabela também estão presentes outras espécies de plantas registradas em observações esporádicas, aumentando os valores para 53 espécies de plantas em 23 famílias. As famílias com maior número de representantes foram Compositae (10 espécies +1 espécie esporádica), Gramineae (no mínimo, 10 espécies) e Leguminosae (6 espécies).

Há cerca de 200 espécies de plantas nos campos de dunas da Praia da Joaquina (Prof. Daniel B. Falkenberg, Departamento de Botânica, Universidade Federal de Santa Catarina, comunicação pessoal) e, portanto, as colônias de Acromyrmex striatus potencialmente forrageam sobre $25 \%$ das espécies de plantas disponíveis.

Revista Brasileira de Zoologia 22 (2): 372-382, junho 2005 
Em 1997, houve uma diminuição tanto no total de plantas forrageadas quanto na riqueza de espécies de plantas no período do outono, talvez em função do grande número de ninhos somente em atividades de limpeza ou desobstrução de galerias (27 em 50 ninhos registrados). Em 1998 houve uma diminuição, no período entre outono e inverno, tanto no total de plantas forrageadas quanto na riqueza de espécies de plantas (Tab. I).

Em 1997, as cinco espécies ou grupos de espécies mais usadas foram: Stylosanthes viscosa Swartz (Leguminosae, 89 registros), Noticastrum malmei Zardini (Compositae, 40), Oxypetalum cf. banksii R. \& S. (Asclepiadaceae, 27), Gramineae não identificadas (25) e Paepalanthus polyanthus (Bong.) Kunth (Eriocaulaceae, 20).

Em 1998, as cinco espécies com maiores números de registros foram: Stylosanthes viscosa (79), Noticastrum malmei (59), Vitex megapotamica (Spreng.) Mold. (Verbenaceae, 30), Paspalum arenarium Schrad. (Gramineae, 25) e Oxypetalum cf. banksii (17).

De modo geral, há um grande número de espécies de plantas pouco utilizadas e um pequeno número de espécies com intenso forrageamento (Tab. I).

As operárias de Acromyrmex striatus podem utilizar tanto material fresco recém cortado, como recolher material seco e caído. A maior parte do material forrageado apresentava porte herbáceo ou prostrado e, mesmo quando arbustivo, as operárias não subiam muito alto nas plantas. Não foi observado forrageamento além de $50,00 \mathrm{~cm}$ do solo.

As figuras 1 e 2 mostram os itens forrageados nos anos de 1997 e 1998, respectivamente. Em 1997, o que mais chama a atenção é a diminuição no uso de folhas verdes no outono e o aumento no uso de fezes de lagartas de Lepidoptera no outono e inverno (Fig. 1). No segundo ano, também no outono, houve uma grande redução no uso de folhas verdes e um aumento no uso de folhas secas. Os valores de porcentagem de utilização de fezes de Lepidoptera se mantiveram relativamente constantes ao longo de 1998 (Fig. 2).

Nos dois anos amostrados, a utilização de recursos por parte de Acromyrmex striatus teve relação com o estado fenológico das plantas ao longo das estações dos anos (teste $\mathrm{G}$ $30,13>\chi^{2} 0,05[3]=7,81$ em 1997; teste $G 40,43>\chi^{2} 0,05[3]=$ 7,81 em 1998).

Em 1997, os valores mostram que há diferenças no uso de plantas em flor e fruto (teste $G 7,09>\chi^{2} 0,10[3]=6,25$ ), já que houve maior coleta de material em flor que em fruto. Por outro lado, em 1998, apesar de as operárias coletarem um pouco mais flores que frutos, o teste não detectou esta diferença (teste G 3,19< $\chi^{2} 0,10[3]=6,25$ ).

Considerando-se o total de itens utilizados ao longo dos anos de 1997 e 1998, encontram-se valores de diversidade de Simpson muito semelhantes (0,89 e 0,90, respectivamente), o que sugere que as amostragens de plantas usadas nesses dois anos provêm de agregações vegetais com a mesma diversidade.

Os valores do teste $t$ mostram que há diferenças entre as
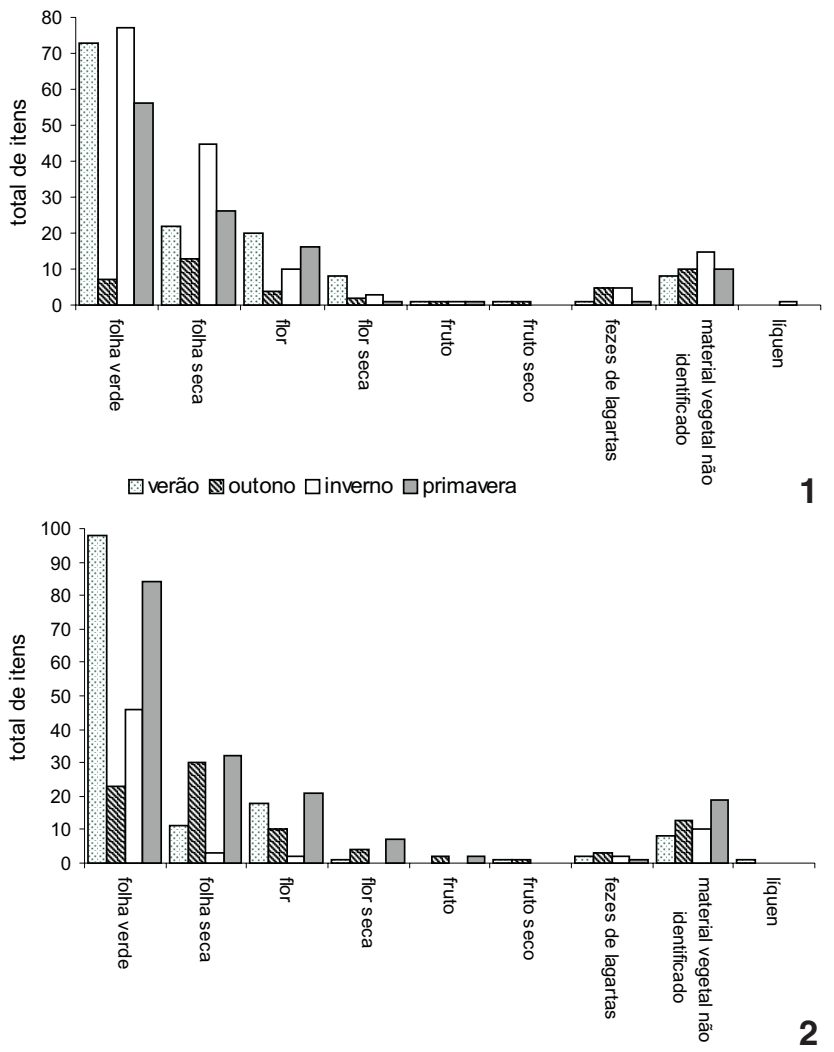

Figuras 1-2. Identificação do material forrageado por Acromyrmex striatus em (1) 1997 e (2) 1998, Praia da Joaquina, Florianópolis, Santa Catarina.

comunidades de plantas usadas por Acromyrmex striatus entre primavera e outono e entre primavera e inverno em 1997 e entre primavera e outono em 1998 (Tab. II).

O índice de similaridade de Morisita para valores anuais de 1997 e 1998 é de 0,96 ou, em outras palavras, as comunidades de plantas usadas por Acromyrmex striatus nos dois anos estudados são idênticas em 96\%. De modo geral, todos os valores foram altos, variando entre 0,76 e 0,98 (Tab. III).

\section{DISCUSSÃO}

Considerando o impacto causado sobre a vegetação, todas as espécies dos gêneros Acromyrmex e Atta e algumas espécies de Trachymyrmex Forel, 1893 podem ser chamadas de polífagas (Pintera 1983, Powell \& Stradling 1991) ou herbívoras generalistas (Rockwood 1977). No entanto, em termos nutricionais, têm-se que diferenciar as larvas e a rainha, que se alimentam do fungo simbionte, sendo exclusivamente micófagas, das operárias, que se alimentam mais de seiva das plantas cortadas e somente um pouco do fungo, sendo então principalmente fitófagas e muito pouco micófagas (Quinlan \& CHERretT 1979, Stradling 1991, Bass \& Cherrett 1995). 
Tabela I. Recursos utilizados por Acromyrmex striatus na Praia da Joaquina, Florianópolis, Santa Catarina, em 1997 e 1998. Número de vezes em que a planta foi utilizada, por estação do ano, sendo: $(V)$ verão, $(O)$ outono, $(I)$ inverno, $(P)$ primavera e $(T)$ total anual. $\left({ }^{*}\right)$ Espécies registradas além do período de observação.

Recursos utilizados

\begin{tabular}{|c|c|c|c|c|c|c|c|c|c|}
\hline \multicolumn{5}{|c|}{1997} & \multicolumn{5}{|c|}{1998} \\
\hline V & $\mathrm{O}$ & 1 & $P$ & $\mathrm{~T}$ & V & $\mathrm{O}$ & 1 & $P$ & $T$ \\
\hline
\end{tabular}

Monocotyledoneae

Cyperaceae

Androtrichum trigynum (Spreng.) Pfeiffer

Cyperus sp.

Scyrpus cernuus Vahl *

Ciperácea indeterminada (pedaço seco)

Eriocaulaceae

Paepalanthus polyanthus (Bong.) Kunth

Gramineae

Cenchrus sp.

Ischaemum minus Presl

Panicum sp. 1

Panicum sp. 2

Paspalum arenarium Schrad.

Paspalum sp.

Rhynchelytrum repens (Willdenow) Hubbard

Sacciolopis sp.

Setaria geniculata (Lam.) Beauv.

Gramíneas indeterminadas

Orchidaceae

Epidendrum fulgens Brongn.

Dicotyledoneae

Asclepiadaceae

Oxypetalum cf. banksii R. \& S.

Chenopodiaceae

Chenopodium sp.

Compositae

Achyroclyne cf. satureioides (Lam.) DC.

Baccharis microcephala (Less.) DC.

Baccharis radicans DC.

Eupatorium casarettoi (Rob.) Steyerm.

Eupatorium sp. *

Gamochaeta sp.

Noticastrum malmei Zardini

Porophyllum ruderale (Jacq.) Cass.

Senecio brasiliensis Less.

Composta indeterminada (pedaço seco)

Convolvulaceae

Ipomoea imperati (Vahl) Grisebach

Ericaceae

Gaylussacia brasiliensis (Spreng.) Meissn.

\begin{tabular}{|c|c|c|c|c|c|c|c|c|c|}
\hline- & - & - & - & - & - & - & - & 1 & 1 \\
\hline 1 & - & - & - & 1 & - & - & - & - & - \\
\hline- & - & 1 & - & 1 & - & - & - & - & - \\
\hline 5 & 2 & 7 & 6 & 20 & 1 & 1 & 5 & 4 & 11 \\
\hline 1 & - & - & - & 1 & - & - & - & - & - \\
\hline- & - & 1 & - & 1 & - & - & 1 & - & 1 \\
\hline- & - & - & 1 & 1 & - & - & - & - & - \\
\hline 3 & - & - & - & 3 & - & - & - & - & - \\
\hline- & - & 2 & 6 & 8 & 11 & 1 & 6 & 7 & 25 \\
\hline 3 & - & 10 & - & 13 & - & - & - & - & - \\
\hline- & - & - & - & - & 1 & - & - & - & 1 \\
\hline 2 & - & - & - & 2 & - & - & - & - & - \\
\hline- & - & - & - & - & 1 & - & - & - & 1 \\
\hline- & 1 & 21 & 3 & 25 & 4 & - & 4 & 7 & 15 \\
\hline 1 & - & - & 1 & 2 & 3 & - & - & 5 & 8 \\
\hline 6 & 2 & 13 & 6 & 27 & 5 & 6 & 3 & 3 & 17 \\
\hline- & - & - & - & - & - & 1 & - & - & 1 \\
\hline- & - & - & - & - & 1 & 1 & - & - & 2 \\
\hline- & - & - & - & - & - & - & - & 1 & 1 \\
\hline- & - & 1 & - & 1 & 5 & 1 & - & 4 & 10 \\
\hline- & - & - & - & - & - & 2 & - & 1 & 3 \\
\hline 2 & - & 1 & 1 & 4 & - & - & 3 & 1 & 4 \\
\hline 16 & 2 & 13 & 9 & 40 & 22 & 10 & 7 & 20 & 59 \\
\hline- & - & - & - & - & - & 2 & - & 1 & 3 \\
\hline- & - & 1 & - & 1 & - & - & - & 1 & 1 \\
\hline- & 1 & 1 & - & 2 & - & - & - & - & - \\
\hline 5 & - & - & 2 & 7 & 4 & 5 & - & 3 & 12 \\
\hline- & 1 & - & - & 1 & - & - & 1 & - & 1 \\
\hline
\end{tabular}


Tabela I. Recursos utilizados por Acromyrmex striatus na Praia da Joaquina, Florianópolis, Santa Catarina, em 1997 e 1998. Número de vezes em que a planta foi utilizada, por estação do ano, sendo: (V) verão, (O) outono, (I) inverno, $(P)$ primavera e (T) total anual. ( $\left.{ }^{*}\right)$ Espécies registradas além do período de observação.

\begin{tabular}{|c|c|c|c|c|c|c|c|c|c|c|}
\hline \multirow{2}{*}{ Recursos utilizados } & \multicolumn{5}{|c|}{1997} & \multicolumn{5}{|c|}{1998} \\
\hline & $\mathrm{V}$ & $\mathrm{O}$ & 1 & $\mathrm{P}$ & $\mathrm{T}$ & $\mathrm{V}$ & $\mathrm{O}$ & 1 & $P$ & $\mathrm{~T}$ \\
\hline \multicolumn{11}{|l|}{ Euphorbiaceae } \\
\hline Sebastiania corniculata (Vahl) Pax & 1 & - & - & - & 1 & 2 & 2 & - & 4 & 8 \\
\hline \multicolumn{11}{|l|}{ Halorrhagaceae * } \\
\hline \multicolumn{11}{|l|}{ Laurembergia tetrandra (Schott) Kanitz * } \\
\hline \multicolumn{11}{|l|}{ Leguminosae } \\
\hline Canavalia rosea (Sw.) DC. & - & - & - & - & - & - & - & - & 1 & 1 \\
\hline Dalbergia ecastophylla (L.) Taub. & - & - & - & - & - & - & - & - & 3 & 3 \\
\hline Desmodium adscendens (Sw.) DC. & - & - & 1 & 2 & 3 & - & - & - & - & - \\
\hline Desmodium barbatum (L.) Benth. & 1 & - & 1 & 1 & 3 & - & - & - & 1 & 1 \\
\hline Indigofera sabulicola Benth. & - & - & - & 1 & 1 & 2 & - & - & 3 & 5 \\
\hline Stylosanthes viscosa Swartz & 28 & 8 & 37 & 16 & 89 & 27 & 19 & 11 & 22 & 79 \\
\hline \multicolumn{11}{|l|}{ Malvaceae } \\
\hline Sida cf. rhombifolia L. & - & - & - & 1 & 1 & - & - & - & - & - \\
\hline \multicolumn{11}{|l|}{ Melastomataceae } \\
\hline Tibouchina urvilleana (DC.) Cogn. & 7 & 4 & 1 & 1 & 13 & 2 & - & - & 2 & 4 \\
\hline Melastomatácea indeterminada (pedaço seco) & - & - & 1 & - & 1 & - & - & - & - & - \\
\hline \multicolumn{11}{|l|}{ Menyanthaceae } \\
\hline Nymphoides indica (L.) Kuntze & - & - & - & 1 & 1 & - & - & - & - & - \\
\hline \multicolumn{11}{|l|}{ Ochnaceae } \\
\hline Sauvagesia erecta $\mathrm{L}$. & - & - & - & - & - & - & - & - & 1 & 1 \\
\hline \multicolumn{11}{|l|}{ Onagraceae } \\
\hline Oenothera mollissima L. & 2 & - & - & 1 & 3 & 1 & - & - & 1 & 2 \\
\hline \multicolumn{11}{|l|}{ Polygalaceae } \\
\hline Polygala cyparissias St.-Hil. \& Moquin & 1 & - & 1 & 1 & 3 & - & 2 & - & 3 & 5 \\
\hline \multicolumn{11}{|l|}{ Rubiaceae } \\
\hline Diodia apiculata (R. \& S.) Schumann & 1 & - & 2 & 3 & 6 & 4 & 3 & 1 & 2 & 10 \\
\hline Richardia sp. & 1 & - & - & - & 1 & - & - & 2 & 1 & 3 \\
\hline \multicolumn{11}{|l|}{ Sapindaceae } \\
\hline Dodonaea viscosa (L.) Jacq. & 1 & - & - & 1 & 2 & - & - & 1 & - & 1 \\
\hline \multicolumn{11}{|l|}{ Solanaceae } \\
\hline Petunia litorallis Smith \& Downs & 2 & - & - & 10 & 12 & 1 & 1 & - & 2 & 4 \\
\hline \multicolumn{11}{|l|}{ Umbelliferae } \\
\hline Centella asiatica (L.) Urb. & - & - & 1 & - & 1 & - & - & 3 & - & 3 \\
\hline Hydrocotyle bonariensis Lam. & 1 & - & - & - & 1 & - & - & 1 & - & 1 \\
\hline Verbenaceae & & - & & & & & & & & \\
\hline Vitex megapotamica (Spreng.) Mold. & 8 & - & 4 & 7 & 19 & 17 & 1 & - & 12 & 30 \\
\hline Material vegetal não identificado & 8 & 10 & 15 & 10 & 43 & 8 & 13 & 10 & 19 & 50 \\
\hline Líquen & - & - & 1 & - & 1 & 1 & - & - & - & 1 \\
\hline Fezes de lagartas de Lepidoptera & 1 & 5 & 5 & 1 & 12 & 2 & 3 & 2 & 1 & 8 \\
\hline Número de espécies & 25 & 10 & 24 & 24 & 41 & 22 & 18 & 16 & 30 & 40 \\
\hline Total de registros & 108 & 36 & 142 & 92 & 378 & 125 & 74 & 61 & 137 & 397 \\
\hline
\end{tabular}

Revista Brasileira de Zoologia 22 (2): 372-382, junho 2005 
Tabela II. Valores de diversidade de Simpson para os recursos usados por Acromyrmex striatus nos anos de 1997 e 1998, Praia da Joaquina, Florianópolis, Santa Catarina. $\left({ }^{*}\right)$ Teste t significativo a 0,05\%.

\begin{tabular}{|c|c|c|c|c|c|c|c|c|}
\hline & \multicolumn{4}{|c|}{1997} & \multicolumn{4}{|c|}{1998} \\
\hline & Verão & Outono & Inverno & Primavera & Verão & Outono & Inverno & Primavera \\
\hline $\begin{array}{l}\text { Índice de Simpson } \\
\text { Teste t }\end{array}$ & 0,88 & 0,83 & 0,87 & 0,91 & 0,88 & 0,87 & 0,89 & 0,91 \\
\hline Verão & - & 1,57 & 0,56 & 1,45 & - & 0,69 & 0,62 & 1,69 \\
\hline Outono & & - & 1,26 & $2,64^{*}$ & & - & 1,20 & $2,06^{\star}$ \\
\hline Inverno & & & - & $2,40 *$ & & & - & 0,96 \\
\hline
\end{tabular}

Tabela III. Valores de similaridade de Morisita para os recursos usados por Acromyrmex striatus nos anos de 1997 e 1998, Praia da Joaquina, Florianópolis, Santa Catarina.

\begin{tabular}{|c|c|c|c|c|c|c|c|c|}
\hline \multirow{2}{*}{ Estações } & \multicolumn{3}{|c|}{1997} & \multicolumn{5}{|c|}{1998} \\
\hline & Verão & Outono & Inverno & Primavera & Verão & Outono & Inverno & Primavera \\
\hline Verão & - & 0,79 & 0,88 & 0,92 & - & 0,88 & 0,85 & 0,98 \\
\hline Outono & & - & 0,82 & 0,76 & & - & 0,94 & 0,93 \\
\hline Inverno & & & - & 0,85 & & & - & 0,81 \\
\hline
\end{tabular}

STRADLING (1991) salienta que o número de espécies de plantas usadas por cortadeiras parcialmente reflete a riqueza de espécies do habitat. No presente caso, uma comparação com outros trabalhos que registram a porcentagem de uso de plantas fica dificultada, uma vez que estes são com diferentes espécies de Atta e em florestas tropicais primárias ou em processos de sucessão secundária. Para Acromyrmex, os poucos estudos que registram porcentagens de uso de plantas são, ou em condições experimentais em laboratório (KNAPP et al. 1990) ou em um bosque subtropical seco, que sofreu cortes seletivos (FARJi-Brenter \& Protomastro 1992). Mesmo para Atta, os valores de uso de plantas são bastante variáveis: Atta cephalotes usa de 22,00\% (RockWood 1976) a 59,00\% (СHerRetT 1968), em função da área estudada e do tempo de coleta dos dados; Atta colombica Guérin, 1845: 28,40\% (SHEPHERD 1985) a 31,40\% (RockWOOD 1976); Atta insularis Guérin, 1845: 52,00\% (PINTERA 1983) e Atta laevigata (Fr. Smith, 1858): 86,00\% (VASCONCElos 1997). Além disso, a contribuição relativa das espécies de plantas pode ser maior para uma espécie de formiga em diferentes habitats, do que para diferentes formigas nos mesmos habitats (VAsCONCELos \& Fowler 1990).

Um outro aspecto importante a ser destacado é que as operárias podem cortar ou experimentar um grupo grande de espécies de plantas e forragear sobre um subgrupo menor dentre as experimentadas (VASCONCELOS \& Fowler 1990). Um bom exemplo é visto em Rockwood (1976), onde colônias de Atta cephalotes e de Atta colombica cortaram folhas de $77,00 \%$ e $67,00 \%$ das espécies de plantas na área estudada, mas só forragearam em $22,00 \%$ e $31,40 \%$ dessas mesmas espécies, respectivamente.

No presente estudo, Acromyrmex striatus experimentou cerca de $25,00 \%$ das plantas presentes nos campos de dunas da Praia da Joaquina, mas forrageou mais fortemente sobre um pequeno grupo de espécies (Tab. I).

Considerando-se as espécies ou grupos de espécies mais forrageadas em 1997 e 1998 por Acromyrmex striatus, nota-se que há preferências tanto por monocotiledôneas (Gramineae não identificadas, Paepalanthus polyanthus e Paspalum arenarium) quanto por dicotiledôneas (Noticastrum malmei, Oxypetalum cf. banksii, Stylosanthes viscosa e Vitex megapotamica). No mesmo local do presente estudo, Castellani et al. (1995b) registraram que Acromyrmex striatus é o principal herbívoro de indivíduos jovens de Paepalanthus polyanthus.

Esses dados são coerentes com as classificações de GoNÇalves (1961), Fowler (1979a), Fowler \& Claver (1991) e FarJIBrener \& Ruggiero (1994) de que Acromyrmex striatus seja uma cortadeira de gramíneas e dicotiledôneas. Diferenças florísticas regionais ou graus de perturbações ambientais podem explicar propostas alternativas de que Acromyrmex striatus forragearia apenas em monocotiledôneas (Fowler et al. 1986, 1989). Por último, segundo proposta de Fowler \& Claver (1991), Acromyrmex striatus poderia ser classificada como uma espécie "pastejadeira" ("grazer"), isto é, que corta gramíneas ou material herbáceo baixo, em níveis moderados em habitats abertos. Além dessa classificação, sugiro que Acromyrmex striatus possa ser encaixada também na categoria de oportunista, segundo os mesmos autores, pois pode também utilizar material vegetal seco e caído. Em um trabalho comparativo na Argentina, Acromyrmex striatus apresentou estratégias de forrageamento mais oportunistas do que Acromyrmex hispidus Santschi, 1925, ao acompanhar as ofertas de espécies de plantas e/ou recursos disponíveis ao longo do ano (Farji-Brener \& Protomastro 1992).

O padrão de muitas espécies de plantas pouco forrageadas e poucas espécies muito forrageadas também está presente em 
Acromyrmex octospinosus (Reich, 1793) (THERRIEN et al. 1986) e em algumas espécies de Atta, a saber: Atta cephalotes (RocKwood 1976, 1977, Berish 1986, Vasconcelos 1990b), Atta colombica (Rockwood 1976, 1977), Atta insularis (Pintera 1983), Atta laevigata (VASCONCELOS 1997) e Atta sexdens (Linnaeus, 1758) (VASCONCElos 1990b). Geralmente os estudos dão mais destaque às espécies de plantas muito utilizadas, mas Fowler et al. (1991) recomendam que se deve também relacionar esse grande número de espécies pouco usadas com os requerimentos nutricionais do fungo simbionte.

Nos cordões de dunas da Praia da Joaquina, as famílias de plantas melhor representadas nas amostragens de Acromyrmex striatus, seja em riqueza de espécies como em freqüência de ataques foram Compositae, Gramineae e Leguminosae. Apesar das dificuldades de comparação, há mais citações para Leguminosae em regiões de mata tropical (Atta cephalotes, CHERRETT 1968, Rockwood 1976, Hubbell et al. 1984 e Atta colombica, Rockwood 1976), enquanto que, em ambientes não florestais, sejam pastagens ou áreas em processos de sucessão secundária, pode haver predomínio de Gramineae ou um equilíbrio no uso de espécies destas três famílias botânicas (Acromyrmex landolti Forel, 1884, WeBER 1947 apud WeBer 1982; Acromyrmex lundi pubescens Emery, 1905, Fowler \& Cabello 1978; Atta cephalotes, Blanton \& EwEL 1985; Atta vollenweideri Forel, 1893, Carvalho \& Tarragó 1982). Leguminosae também foi a família de planta com maior taxa de visitas por diversas espécies de formigas, em um estudo que quantificou o uso de recursos líquidos provenientes de plantas e acessíveis a uma comunidade de formigas em região costeira, no México (Rico-Gray 1993).

Além destas, duas outras famílias merecem comentários: Asclepiadaceae e Orchidaceae. A primeira é caracterizada pela presença de vasos laticíferos e teve a espécie Oxypetalum cf. banksii como uma das mais utilizadas por Acromyrmex striatus. As formigas cortavam, em proporções semelhantes, folhas e flores, frescas ou secas, mesmo com a adesão de peças bucais, antenas e pernas pelo látex liberado no corte de material fresco. Em outras palavras, não houve preferências pelo corte de material seco, já sem látex, seja numa escala momentânea ou sazonal. Estes dados são contrastantes com os de STRAdLing (1978) e Hubbell et al. (1984), que apontam diferenças nas taxas de ataques em plantas com látex, ou em escala sazonal ou experimentalmente, com folhas cortadas e secas. Quanto à família Orchidaceae, houve apenas uso de flores frescas ou secas de Epidendrum fulgens Brongn. No trabalho de SHEPHERD (1985), Atta colombica também não cortou folhas de espécies de Orchidaceae.

A diminuição no uso de folhas verdes e aumento no uso de folhas secas, registrado no outono, para os ninhos de Acromyrmex striatus, nos dois anos de observação, parece indicar uma mudança sazonal no uso destes recursos. Alguns estudos têm mostrado haver diferenças sazonais no uso de material vegetal fresco ou seco, geralmente relacionadas a mudanças climáticas: Attini “inferiores" (Leal \& Oliveira 1998); Acromyrmex hispidus e Acromyrmex striatus [FarJi-Brener \& Protomastro
(1992); Acromyrmex octospinosus, WetTerer (1991); Acromyrmex subterraneus subterraneus Forel, 1893, Maciel et al. (1995); Acromyrmex volcanus Wheeler, 1937, Wetterer (1993); Atta cephalotes, Rockwood (1975, 1976), Vasconcelos (1990b); Atta colombica, Rockwood (1975, 1976), Wirth et al. (1997); Atta insularis, Perez-Perera et al. (1990); Atta laevigata, Vasconcelos \& Cherrett (1996) e Atta sexdens, Vasconcelos (1990b)]. Wirth et al. (1997) destacam que deve ser dada mais importância ao material vegetal não verde e que este geralmente não é quantificado ou considerado em estimativas anuais de forrageamento. VAsconcelos \& Cherrett (1996) dizem que a preferência por folhas secas pode ser devida ao aumento na concentração de nutrientes, a diferenças na quantidade de água e diminuição na efetividade ou na concentração de substâncias repelentes. Uma outra possível função desse material vegetal seco trazido ao ninho é o de absorver o excesso de umidade da colônia (SCHADE 1973 apud Fowler 1979b).

O uso de fezes de lagartas de Lepidoptera como substrato para a fungicultura, embora não tenha tido valores expressivos nos dois anos, sempre foi um recurso disponível para Acromyrmex striatus no presente trabalho. WeTterer et al. (1998) registraram até 11,00\% de cargas com fezes de lagartas em uma população de Acromyrmex octospinosus e argumentam que esse valor expressivo pode ser devido a uma explosão populacional de lagartas no período estudado. Estes autores sugerem ainda que, embora fezes de insetos sejam um recurso geralmente usado por atíneos "inferiores", este não é um tipo de recurso produzido em grande escala em termos temporais e espaciais, suficiente para suportar grandes colônias, como é o caso das colônias de Acromyrmex e de Atta.

Uma das diferenças básicas na ecologia de forrageamento das cortadeiras é que, enquanto algumas espécies de Atta forrageiam quase que exclusivamente sobre matéria vegetal recém cortada de árvores (por exemplo, Atta cephalotes, VAsCONCELos 1990b), diversas espécies de Acromyrmex utilizam matéria vegetal fresca cortada de ervas e arbustos, além de material vegetal seco e caído, sejam folhas, flores e frutos, podendo se comportar não como cortadeiras exclusivas, mas como cortadeiras e oportunistas (Farji-Brener \& Protomastro 1992, Diehl-Fleig 1995, Wetterer et al. 1998).

As inter-relações entre parâmetros físicos e químicos ajudam a entender um dos aspectos do forrageamento em cortadeiras que é o de cortar ou não folhas jovens. Assim, enquanto os trabalhos de Cherrett (1972), LitTledyke \& Cherrett (1978), WALLER (1982a, b) e BeRISH (1986) apontam para preferência por folhas jovens, relacionando suculência e ausência de substâncias repelentes, os trabalhos de Morini et al. (1993) e de Fowlen \& SCHLINDWEIN (1994) sugerem a preferência por folhas velhas, relacionando-a com a evaporação de substâncias repelentes nestas folhas velhas. LitTLEDyKe \& CHeRReTt (1978) chegam a sugerir que, talvez folhas jovens sejam mais importantes para as operárias, pela ingestão direta da seiva das plantas, enquanto que folhas velhas seriam mais importantes para o fungo simbionte. 
$\mathrm{O}$ aspecto da abundância das plantas herbivoradas por cortadeiras também tem chamado a atenção dos pesquisadores. BlANTON \& EwEl (1985) e VASCONCELOS (1997) encontraram relações positivas entre a abundância das plantas preferidas e a taxa de herbivoria, enquanto que Rockwood (1976) não aponta para uma relação direta entre plantas mais abundantes e maior taxa de ataque. Talvez a abundância explique, ao menos em parte, a preferência das cortadeiras. Na Praia da Joaquina, a escolha das plantas para corte por parte de Acromyrmex striatus parece estar relacionada à abundância e ocorrência das espécies nas diferentes regiões dos cordões arenosos estudados. Assim, na região de duna frontal, mais voltada para o mar, geralmente as plantas mais herbivoradas nessa região (relacionadas em seqüência de importância de herbivoria: Oxypetalum cf. banksii, Ipomoea imperati (Vahl) Grisebach, Petunia littoralis Smith \& Downs, Polygala cyparissias St.-Hil. \& Moquin, Oenothera molissima L., Porophyllum ruderale (Jacq.) Cass., Hydrocotyle bonariensis Lam. e Canavalia rosea (Sw.) DC.) são também aquelas com maiores freqüências na duna frontal na Praia da Joaquina (CASTELLANI et al. 1999). Também, em observações em regiões mais para o interior, posterior ao setor de dunas frontais, as maiores taxas de herbivoria correspondem às espécies mais freqüentes nesses locais (relacionadas em seqüência de importância de herbivoria: Stylosanthes viscosa, Noticastrum malmei, Paepalanthus polyanthus e Paspalum arenarium) (CASTELLANi et al. 1995a, 1996).

O que se pretende frisar é que, somente abundância ou ausência/presença de substâncias químicas parecem não ser suficientes para tentar explicar a preferência por esta ou aquela espécie de planta (Roces 1994). Todos esses aspectos, mais a presença de látex, a interação com formigas agressivas nas plantas, as necessidades internas de cada colônia e a época da produção de sexuados, tudo isso junto compõe um painel complexo do qual vê-se apenas um detalhe ao se registrar operárias cortando partes de uma planta.

Na área estudada, Acromyrmex striatus parece utilizar as mesmas espécies de plantas, mês após mês, somente fazendo pequenas modificações na qualidade e quantidade do material de substrato ao fungo (Tabs II e III). Talvez fosse interessante acompanhar sazonalmente alguns ninhos marcados para uma quantificação mais precisa dessa variação na escolha dos substratos.

Antes de terminar, cabe uma pergunta: Acromyrmex striatus pode vir a se tornar uma espécie praga? Em ambientes não modificados, como o do presente estudo, Acromyrmex striatus corta uma grande variedade de plantas, mas também usa muito material vegetal seco e caído, se comportando como uma cortadeira e oportunista. Porém, em ambientes modificados para pecuária ou agricultura, Acromyrmex striatus pode se utilizar de plantas ou recursos fornecidos pelo Homem, podendo ser considerada espécie praga (GonÇALves 1961, Fowler 1979a, Quirán 1998). Além disso, quando presentes em área de pastagem, podem reduzir em muito a área aproveitável do campo, devido ao tipo de formigueiro com área limpa ao redor das aberturas (GONÇALVEs 1961).
No entanto, é visão corrente que a influência antrópica, ao modificar os ambientes, é que causa o aumento na distribuição de algumas espécies de cortadeiras, bem como altera o padrão de comunidades das espécies preexistentes, favorecendo o aumento no número de espécies consideradas pragas (WEBER 1982, Fowler et al. 1986, 1989, VASCONCELos 1990a, FARJi-Brener \& Ruggiero 1994, Vasconcelos \& Cherrett 1995, Farji-Brener 1996, DieHL-Fleig 1997).

\section{AGRADECIMENTOS}

Ao Prof. Dr. Harold Gordon Fowler, do Instituto de Biociências-UNESP-Rio Claro, pela orientação e à CAPES, pela concessão de bolsa durante o período do Doutorado.

\section{REFERÊNCIAS BIBLIOGRÁFICAS}

Bass, M. \& J.M. Cherrett. 1995. Fungal hyphae as a source of nutrients for the leaf-cutting ant Atta sexdens. Physiological Entomology, London, 20 (1): 1-6.

Berish, C.W. 1986. Leaf-cutting ants (Atta cephalotes) select nitrogen-rich forage. The American Midland Naturalist, Columbus, 115 (2): 268-276.

Blanton, C M. \& J.J. Ewel. 1985. Leaf-cutting ant herbivory in successional and agricultural tropical ecosystems. Ecology, Washington, 66 (3): 861-869.

Bowers, M.A. \& S.D. Porter. 1981. Effect of foraging distance on water content of substrates harvested by Atta colombica (Guerin). Ecology, Washington, 62 (1): 273-275.

Brower, J.E.; J.H. ZAR \& C.N. vON ENDE. 1998. Field \& laboratory methods for general ecology. Boston, WCB McGraw-Hill, $4^{\text {th }}$ ed., XI+273p.

Carvalho, S. \& M.F.S. TARragó. 1982. Atta (Neoatta) vollenweideri Forel, 1893, no Brasil: ocorrência, aspectos externos e internos do sauveiro (Hymenoptera: Formicidae). Revista do Centro de Ciências Rurais, Santa Maria, 12 (1): 1-20.

Castellani, T.T.; R. Folchini \& K.Z. Scherer. 1995a. Variação temporal da vegetação em um trecho de baixada úmida entre dunas, Praia da Joaquina, Florianópolis, SC. Insula, Florianópolis, 24: 37-72.

Castellani, T.T.; K.Z. Scherer; L.M. Locatelli \& B.C. Lopes. 1995b. The occurrence of Junonia evarete (Lepidoptera: Nymphalidae) and Acromyrmex striatus (Hymenoptera: Formicidae) on Paepalanthus polyanthus (Eriocaulaceae). Journal of the New York Entomological Society, New York, 103 (3): 329-334.

Castellani, T.T.; S. Vieira \& K.Z. Scherer. 1996. Contribuição ao conhecimento da distribuição espacial de Paepalanthus polyanthus (Bong.) Kunth (Eriocaulaceae) em áreas de baixada úmida de dunas. Acta Botanica Brasilica, São Paulo, 10 (1): 25-36.

Castellani, T.T.; C.A. Caus \& S. Vieira. 1999. Fenologia de uma comunidade de duna frontal no sul do Brasil. Acta Botanica Brasilica, São Paulo, 13 (1): 99-114. 
Cherrett, J.M. 1968. The foraging behaviour of Atta cephalotes L. (Hymenoptera, Formicidae) I. Foraging pattern and plant species attacked in tropical rain forest. Journal of Animal Ecology, London, 37: 387-403.

Cherrett, J.M. 1972. Some factors involved in the selection of vegetable substrate by Atta cephalotes (L.) (Hymenoptera: Formicidae) in tropical rain forest. Journal of Animal Ecology, London, 41: 647-660.

DieHL-Fleig, E. 1995. Formigas - organização social e ecologia comportamental. São Leopoldo, Editora Unisinos, 166p.

Diehl-Fleig, E. 1997. Ocorrência de Acromyrmex em áreas com distintos níveis de perturbação antrópica no Rio Grande do Sul. Acta Biologica Leopoldensia, São Leopoldo, 19 (2): 165-171.

Farji-Brener, A.G.F. 1996. Posibles vías de expansión de la hormiga cortadora de hojas Acromyrmex lobicornis hacia la Patagonia. Ecología Austral, Luján, 6: 144-150.

Farji-Brener, A.G.F. \& J.J. Protomastro. 1992. Patrones forrajeros de dos especies simpátricas de hormigas cortadoras de hojas (Attini, Acromyrmex) en un bosque subtropical seco. Ecotropicos, Mérida, 5 (1): 32-43.

Farji-Brener, A.G.F. \& A. Ruggiero. 1994. Leaf-cutting ants (Atta and Acromyrmex) inhabiting Argentina: patterns in species richness and geographical range sizes. Journal of Biogeography, Oxford, 21 (4): 391-399.

FarJi-Brener, A.G.F. \& J.F. SiLVA. 1995. Leaf-cutting ants and forest groves in a tropical parkland savanna of Venezuela: facilitated succession? Journal of Tropical Ecology, Cambridge, 11 (4): 651-669.

FarJi-Brener, A.G.F. \& L. Ghermandi. 2000. Influence of nests of leaf-cutting ants on plant species diversity in road verges of northern Patagonia. Journal of Vegetation Science, Uppsala, 11: 453-460.

Farji-Brener, A.G.F. \& A.E. Illes. 2000. Do leaf-cutting ant nests make "bottom-up" gaps in neotropical rain forests?: a critical review of the evidence. Ecology Letters, Paris, 3: 219-227.

Fowler, H.G. 1979a. Las hormigas cortadoras del Paraguay de los géneros Atta Fabricius y Acromyrmex Mayr: bionomíco, distribución y sistemática. Informes Científicos, Universidad Nacional de Asunción, Asunción, 2 (1): 30-70.

Fowler, H.G. 1979b. Environmental correlates of the foraging of Acromyrmex crassispinus. Ciência e Cultura, São Paulo, 31 (8): 879-882.

Fowler, H.G. \& L. Cabello. 1978. Bionomics of Acromyrmex lundi pubescens (Emery) in Paraguay (Hymenoptera: Formicidae). Entomological News, Philadelphia, 89 (7-8): 175-177.

Fowler, H.G. \& E.W. STiles. 1980. Conservative resource management by leaf-cutting ants? The role of foraging territories and trails, and environmental patchiness. Sociobiology, Chico, 5 (1): 25-41.

Fowler, H.G. \& S. Claver. 1991. Leaf-cutter ant assemblies: effects of latitude, vegetation, and behaviour, p. 51-59. In: C.R. Huxley \& D.D. Cutler (Eds). Ant-plant interactions. Oxford,
Oxford University Press, 601p.

Fowler, H.G. \& M.N. Schlindwein. 1994. Influence of natural fragrances on recruitment olfactory conditioning and acceptance of forage material in the leaf-cutting ant Atta sexdens rubropilosa (Hymenoptera: Formicidae). Etología, Barcelona, 4: 27-32.

Fowler, H.G.; L.C. Forti; V. Pereira-da-Silva \& N.B. Saes. 1986. Economics of grass-cutting ants, p. 18-35. In: C. S. LofGren $\&$ R. K. Vander Meer (Eds.). Fire ants and leaf-cutting ants - biology and management. Boulder, Westview Press, 435p.

Fowler, H.G.; M.I. Pagani; O.A. DA Silva; L.C. Forti; V. Pereira-dASilva \& H.L. Vasconcelos. 1989. A pest is a pest is a pest? The dilemma of Neotropical leaf-cutting ants: keystone taxa of natural ecosystems. Environmental Management, New York, 13 (6): 671-675.

Fowler, H.G.; L.C. Forti; C.R.F. Brandão; J.H.C. Delabie \& H.L. VASCONCELOS. 1991. Ecologia nutricional de formigas, p. 131223. In: A.R. PANIZZI \& J.R.P. PARRA (Eds). Ecologia nutricional de insetos e suas implicações no manejo de pragas. São Paulo, Editora Manole \& CNPq, 359p.

Gonçalves, C.R. 1961. O gênero Acromyrmex no Brasil (Hym., Formicidae). Studia Entomologica, Petrópolis, 4 (1-4): 113180.

Hölldobler, B. \& E.O. Wilson. 1990. The ants. Cambridge, The Belknap Press of the Harvard University Press, 732p.

Howard, J.J. 1988. Leafcutting ant diet selection: relative influence of leaf chemistry and physical features. Ecology, Washington, 69 (1): 250-260.

HOWARD, J.J.1990. Infidelity of leafcutting ants to host plants: resource heterogeneity or defense induction? Oecologia, Heidelberg, 82 (3): 394-401.

HowARD, J.J. 1991. Resource quality and cost in the foraging of leaf-cutter ants, p. 42-50. In: C.R. Huxley \& D.D. Cutler (Eds). Ant-plant interactions. Oxford, Oxford University Press, $601 p$.

Hubbell, S.P.; J.J. Howard \& D.F. Wiemer. 1984. Chemical leaf repellency to an attine ant: seasonal distribution among potential host plant species. Ecology, Washington, 65 (4): 1067-1076.

JANZEN, D.H. 1980. Ecologia vegetal nos trópicos. São Paulo, E.P.U., EDUSP, 79p.

KNAPP, J.J.; P.E. Howse \& A. Kermarrec. 1990. Factors controlling foraging patterns in the leaf-cutting ant Acromyrmex octospinosus (Reich), p. 382-409. In: R. K. VANDER MeER; K. Jaffé \& A. Cedeño (Eds). Applied myrmecology - a world perspective. Boulder, Westview Press, 741p.

Leal, I.R. \& P.S. Oliveira. 1998. Interactions between fungusgrowing ants (Attini), fruits and seeds in cerrado vegetation in Southeast Brazil. Biotropica, Washington, 30 (2): 170-178.

Littledyke, M. \& J.M. CherretT. 1978. Defence mechanisms in young and old leaves against cutting by the leaf-cutting ants Atta cephalotes (L.) and Acromyrmex octospinosus (Reich) (Hymenoptera: Formicidae). Bulletin of Entomological 
Research, Cardiff, 68: 263-271.

Maciel, M.A.F.; T.M.C. Della Lucia; M.S. Araújo \& M.A. Oliveira. 1995. Ritmo diário de atividade forrageadora da formiga cortadeira Acromyrmex subterraneus subterraneus Forel. Anais da Sociedade Entomológica do Brasil, Curitiba, 24 (2): 371 378.

Morini, M.S. De C.; H.G. Fowler; F.M. Schlittler \& O.C. Bueno. 1993. Polymorphic and relative pick-up responses of a leafcutting ant, Atta sexdens rubropilosa (Hymenoptera: Formicidae), to extracts from new and old Eucalyptus leaves. Journal of Plant Protection in the Tropics, Kuala Lumpur, 10 (1): 59-62.

Moutinho, P.; D.C. Nepstad \& E.A. Davidson. 2003. Influence of leaf-cutting ant nests on secondary Forest growth and soil properties in Amazonia. Ecology, Washington, 84 (5): 1265 1276.

Perez-Perera, L.F.; A. Gonzalez \& J.F. Martinez. 1990. Seasonal activity of Atta insularis, an important citrus pest in Jaguey Grande, Cuba, p. 242-250. In: R.K. Vander Meer; K. Jaffé \& A. Cedeño (Eds). Applied myrmecology - a world perspective. Boulder, Westview Press, 741p.

Pintera, A. 1983. Selection of plants utilized by Atta insularis in Cuba (Hymenoptera, Formicidae). Acta Entomologica Bohemoslovaca, Ceske Budojovice, 80: 13-20.

Powell, R.J. \& D.J. StRadling. 1991. The selection and detoxification of plant material by fungus-growing ants, p. 19-41. In: C.R. Huxley \& D.F. Cutler (Eds). Ant-plant interactions. Oxford, Oxford University Press, 601p.

Quinlan, R.J. \& J.M. Cherrett. 1979. The role of fungus in the diet of the leaf-cutting ant Atta cephalotes (L.). Ecological Entomology, Carmarthen, 4: 151-160.

QuiráN, E.M. 1998. Hormigas cortadoras en Argentina, p. 5775. In: E. Berti-Filho; F.A.M. Mariconi \& L.R. Fontes (Eds). Anais do simpósio sobre formigas cortadeiras dos países do Mercosul. Piracicaba, FEALQ, 139p.

Rico-Gray, V. 1993. Use of plant-derived food resources by ants in the dry tropical lowlands of coastal Veracruz, Mexico. Biotropica, Washington, 25 (3): 301-315.

Roces, F. 1994. Odour learning and decision-making during food collection in the leaf-cutting ant Acromyrmex lundi. Insectes Sociaux, Leuven, 41 (3): 235-239.

Rockwood, L.L. 1975. The effects of seasonality on foraging in two species of leaf-cutting ants (Atta) in Guanacaste Province, Costa Rica. Biotropica, Washington, 7 (3): 176-193.

Rockwood, L.L. 1976. Plant selection and foraging patterns in two species of leaf-cutting ants (Atta). Ecology, Washington, 57 (1): 48-61.

Rockwood, L.L. 1977. Foraging patterns and plant selection in Costa Rican leaf cutting ants. Journal of the New York Entomological Society, New York, 85 (4): 222-233.

SHEPHERD, J.D. 1985. Adjusting foraging effort to resources in adjacent colonies of the leaf-cutter ant, Atta colombica. Biotropica, Washington, 17 (3): 245-252.
Strading, D.J. 1978. The influence of size on foraging in the ant, Atta cephalotes, and the effect of some plant defence mechanisms. Journal of Animal Ecology, London, 47 (1): 173-188.

STRADLING, D.J. 1991. An introduction to the fungus-growing ants, Attini, p. 15-18. In: C.R. Huxley \& D.F. CuTler (Eds). Ant-plant interactions. Oxford, Oxford University Press, 601p.

Sugayama, R L. \& A. Salatino. 1995. Influence of leaf epicuticular waxes from cerrado species on substrate selection by Atta sexdens rubropilosa. Entomologia Experimentalis et Applicata, Dordrecht, 74 (1): 63-69.

Therrien, P.; J.N. McNeil; W.G. Wellington \& G. Febvay. 1986. Ecological studies of the leaf-cutting ant, Acromyrmex octospinosus, in Guadeloupe, p. 172-183. In: C.S. LOFGren \& R.K. VAnder Meer (Eds). Fire ants and leaf-cutting ants biology and management. Boulder, Westview Press, 435p.

VASCONCELOS, H.L. 1990a. Habitat selection by the queens of the leaf-cutting ant Atta sexdens L. in Brazil. Journal of Tropical Ecology, Cambridge, 6: 249-252.

VASCONCELOS, H.L. 1990b. Foraging activity of two species of leaf-cutting ants (Atta) in a primary forest of the Central Amazon. Insectes Sociaux, Leuven, 37 (2): 131-145.

VASCONCELOS, H.L. 1997. Foraging activity of an Amazonian leafcutting ant: responses to changes in the availability of woody plants and to previous plant damage. Oecologia, Heidelberg, 112: 370-378.

Vasconcelos, H.L. \& H.G. Fowler. 1990. Foraging and fungal substrate selection by leaf-cutting ants, p. 410-419. In: R.K. Vander Meer; K. Jaffé \& A. Cedeño (Eds). Applied myrmecology - a world perspective. Boulder, Westview Press, 741p.

VASCONCELos, H.L. \& J.M. CherRetT. 1995. Changes in leaf-cutting ant populations (Formicidae: Attini) after the clearing of mature forest in Brazilian Amazonia. Studies on Neotropical Fauna and Environment, Lisse, 30 (2): 107-113.

Vasconcelos, H.L. \& J.M. Cherrett. 1996. The effect of wilting on the selection of leaves by the leaf-cutting ant Atta laevigata. Entomologia Experimentalis et Applicata, Dordrecht, 78: 215-220.

Vasconcelos, H.L. \& J.M. Cherrett. 1997. Leaf-cutting ants and early forest regeneration in central Amazonia: effects of herbivory on tree seedling establishment. Journal of Tropical Ecology, Cambridge, 13: 357-370.

Verchot, L.V.; P.R. Moutinho \& E.A. Davidson. 2003. Leaf-cutting ant (Atta sexdens) and nutrient cycling: deep soil inorganic nitrogen stocks, mineralization, and nitrification in Eastern Amazonia. Soil Biology and Biochemistry, Amsterdam, 35 (9): 1219-1222.

WALLER, D.A. 1982a. Leaf-cutting ants and live oak: the role of leaf toughness in seasonal and intraspecific host choice. Entomologia Experimentalis et Applicata, Dordrecht, 32: 146-150.

WALLER, D.A. 1982b. Leaf-cutting ants and avoided plants: defences against Atta texana attack. Oecologia, Heidelberg,

Revista Brasileira de Zoologia 22 (2): 372-382, junho 2005 
52 (3): 400-403.

Weber, N.A. 1972. Gardening ants, the Attines. Memoirs of the American Philosophical Society, Philadelphia, 92: 1146.

Weber, N.A. 1982. Fungus ants, p. 255-363. In: H. R. Hermann (Ed.). Social insects. New York, Academic Press.

Wetterer, J.K. 1991. Foraging ecology of the leaf-cutting ant Acromyrmex octospinosus in a Costa Rican rain forest. Psyche, Cambridge, 98: 361-371.

Wetterer, J.K. 1993. Foraging and nesting ecology of a Costa Rican leaf-cutting ant, Acromyrmex volcanus. Psyche,

Recebido em 05.VIII.2004; aceito em 17.V.2005.
Cambridge, 100 (1-2): 65-76.

Wetterer, J.K.; D.S. Gruner \& J.E. Lopez. 1998. Foraging and nesting ecology of Acromyrmex octospinosus (Hymenoptera: Formicidae) in a Costa Rican tropical dry forest. Florida Entomologist, Gopher, 81 (1): 61-67.

Wirth, R.; W. Beyschlag; R.J. Ryel \& B. Hölldobler. 1997. Annual foraging of the leaf-cutting ant Atta colombica in a semideciduous rain forest in Panama. Journal of Tropical Ecology, Cambridge, 13: 741-757. 